\title{
Ensamble de aves en una mitilicultura de Chiloé, sur de Chile
}

Assemblage of birds in a mussel farm on Chiloé Island, southern Chile

\author{
Jaime A. Cursach ${ }^{1}$, Cristián G. Suazo² ${ }^{2}$ Jaime R. Rau $^{1,3}$, \\ Claudio N. Tobar ${ }^{1}$ y Alberto Gantz ${ }^{1}$
}

\begin{abstract}
${ }^{1}$ Laboratorio de Ecología, Departamento de Ciencias Básicas, Universidad de Los Lagos, casilla 933, Osorno, Chile. jcurval@gmail.com

${ }^{2}$ Instituto de Ciencias Ecologicas y Evolutivas, Facultad de Ciencias, Universidad Austral de Chile, casilla 567, Valdivia, Chile ${ }^{3}$ Programa IBAM, Universidad de Los Lagos, casilla 933, Osorno, Chile
\end{abstract}

\begin{abstract}
We evaluated the composition and seasonal variation in the diversity of an aquatic bird assemblage related to a mussel farm in southern Chile. Observations were carried out during spring 2008, and the summer, autumn and winter of 2009. Neotropic cormorant Phalacrocorax brasilianus, followed by kelp gull Larus dominicanus and imperial cormorant P. atriceps were the dominant species. We identified five dietary groups, of which piscivorous birds had the greatest representation. Finally, we discuss the need to evaluate the potential impacts generated by the intensive mussel farming on various components of the marine ecosystem in southern Chile.
\end{abstract}

Key words: Aquaculture, conservation, seabirds

\section{INTRODUCCIÓN}

La acuicultura se destaca en la actualidad por intentar producir la mayor cantidad de organismos hidrobiológicos en el menor espacio y tiempo posible. Esta conglomeración de individuos genera la atracción de fauna silvestre que busca depredar a los organismos en cultivo y/o sus desechos orgánicos (Roycroft et al. 2004). Por ejemplo en Chile, en la industria salmonera, actividad acuícola con mayor desarrollo en el país, se ha registrado una fuerte asociación de organismos vertebrados que depredan los peces en cultivo, generando discusiones sociales sobre el impacto de esta actividad en la fauna silvestre y los ambientes costeros del sur de Chile (Buschmann et al. 2006).

Después de la salmonicultura, la mitilicultura es la segunda actividad acuícola con mayor producción en Chile $^{1}$. El 99,8\% de la industria nacional durante los años 1999-2009 se desarrolló en el sur del país (región de Los Lagos: $41-43^{\circ} \mathrm{S}$ ), donde la principal especie en cultivo es el bivalvo Mytilus chilensis (Hupe, 1854) ${ }^{1}$. Pese a la elevada tasa intrínsica de crecimiento anual en la producción de esta industria (23,3\%), durante los años 1999 y 2009¹, existe muy poca información sobre los efectos que genera el cultivo intensivo de mitílidos sobre los componentes del ecosistema marino del sur de Chile, de hecho, en ISI Web of Knowledge no se encontraron artículos relacionados a este tópico en Chile. Actualmente, la mayoría de los artículos publicados analizan etapas de desarrollo y optimización en el crecimiento de los mitílidos, pero ninguno de ellos discute las implicancias ecológicas de este cultivo sobre componentes ecosistémicos como la fauna silvestre. Por ello, el objetivo del presente trabajo fue caracterizar la composición y variación estacional del ensamble de aves asociado a un centro mitilicultor del sur de Chile.

\section{MATERIALES Y MÉtodos}

Las observaciones fueron realizadas en el Centro mitilicultor Putemún (42²7'29,31'’s; 7344’53,49”'O) ubicado al interior de la Reserva Marina Putemún, en el extremo norte del fiordo de Castro, isla grande de Chiloé, sur de Chile. El Centro Putemún estuvo compuesto por dos líneas flotantes dobles de $100 \mathrm{~m}$ de largo cada una, que en conjunto abarcó un área de $340 \mathrm{~m}^{2}$, proporcionando una biomasa de ca. 10 ton de bivalvos mitílidos, e.g., chorito y choro zapato Choromytilus chorus (Molina, 1782) en estado juvenil ( 3 a $4 \mathrm{~cm}$ de longitud de valva),

\footnotetext{
${ }^{1}$ \ttp:// www. sernapesca.cl/ index. php?option=com_remository\&ltemid=54\&func=select\&id=2>
} 
acompañado de pequeñas concentraciones de cholga Aulacomya atra (Molina, 1782). Durante los meses de septiembre a diciembre (primavera) de 2008, enero y febrero (verano), abril y junio (otoño), julio y agosto (invierno) de 2009, se realizaron registros quincenales de la riqueza y abundancia de las aves marinas asociadas al Centro Putemún. Las observaciones se realizaron desde un punto fijo (Bibby et al. 2000) por un período de $10 \mathrm{~min}$, entre las 09:00 y 13:00 h. Este punto de observación permitió una visión total del área de estudio $\left(340 \mathrm{~m}^{2}\right)$, desde una distancia de $60 \mathrm{~m}$, apoyado por el uso de binoculares ( $10 \times 50)$. Todos los censos se realizaron en días despejados y sin viento fuerte, totalizando $200 \mathrm{~h}$ de observación.

Para la clasificación taxonómica de las aves, se utilizó la lista del 'South American Classification Commitee' (Remsen et al. 2011). Posteriormente, se clasificó a cada especie dentro de un grupo alimenticio, siguiendo las revisiones de Arriagada (2004), Jaksic (2004), Vilina et al. (2006). La riqueza específica mensual observada correspondió al número total de especies registradas en cada mes, en tanto, la riqueza específica mensual esperada fue calculada mediante rarefacción con el programa Biodiversity Professional Beta 1 (McAleece et al. 1997). La abundancia relativa mensual de cada especie se estableció mediante el promedio de individuos registrados para cada mes. La abundancia relativa del total de individuos observados se definió como la suma de las abundancias mensuales de todas las especies. Los cambios mensuales en la abundancia de aves fueron evaluados mediante la prueba no paramétrica de KruskalWallis $(\mathrm{H})$, con un nivel de significancia estadística de 0,05 .

\section{Resultados y Discusión}

Durante el período de estudio se observó un total de 2583 aves pertenecientes a 17 especies y nueve familias (Tabla 1). Laridae presentó la mayor riqueza, seguida por Phalacrocoracidae (Tabla 1). El yeco Phalacrocorax brasilianus (Gmelin, 1789) presentó el mayor valor de abundancia relativa total con 1768 individuos, seguido por la gaviota dominicana Larus dominicanus (Lichtenstein, 1823) y el cormorán imperial Phalacrocorax atriceps (King, 1828) (Tabla 1). La riqueza específica mensual varió entre siete y diez especies. La mayor riqueza se registró en la temporada primaveral 2008 y la menor durante otoño e invierno 2009 (Tabla 1). A su vez, se obtuvo una alta concordancia entre los valores mensuales de riqueza observada y esperada por rarefacción, lo que se atribuye a la alta abundancia de individuos registrados (rango: 152 a 464 individuos mes ${ }^{-1}$, Tabla 1). Los valores mensuales de abundancia relativa de individuos no presentaron diferencias estadísticamente significativas $(\mathrm{H}=0,437$, g.l. $=9, P>0,05$, Tabla 1$)$, sin embargo, la mayor abundancia se registró durante la temporada invernal 2009 y la mínima en primavera 2008 (Tabla 1).

Se identificaron cinco grupos alimenticios para la totalidad de las aves presentes. El 71,3\% de la abundancia relativa del total de individuos fueron aves piscívoras, mientras que el $18,4 \%$ perteneció al grupo de aves depredadoras de peces e invertebrados. El 10\% correspondió a aves omnívoras y el 0,3\% restante fue compartido por los grupos alimenticios de aves depredadoras de invertebrados y aves herbívoras.

El ensamble de aves estuvo dominado por pocas especies muy abundantes de las familias Phalacrocoracidae y Laridae. Este tipo de resultado es similar a lo observado en las costas de Irlanda, donde también se ha constatado que especies pertenecientes a estas dos familias son relevantes para el ensamble de aves asociadas a centros de mitilicultura (Roycroft et al. 2004). El yeco, seguido por la gaviota dominicana y el cormorán imperial, presentaron los mayores valores de abundancia relativa total, por lo cual fueron consideradas como las especies dominantes del ensamble de aves presentes en el Centro Putemún.

La dieta del yeco se compone principalmente de peces de tamaño pequeño que captura buceando en aguas poco profundas (Quintana et al. 2004, González-Gajardo et al. 2009). Mientras que la gaviota dominicana es una especie generalista, su dieta registra peces, invertebrados y una constante tendencia a la explotación de recursos de origen humano (Bertellotti \& Yorio 1999, Silva et al. 2000, Petracci et al. 2004, Ludynia et al. 2005). La dieta del cormorán imperial se compone preferentemente de peces pequeños, así como también de moluscos cefalópodos y crustáceos (Casaux et al. 1997, Gosztonyi \& Kuba 1998, Ferrari et al. 2004). Por lo anterior, estas tres especies dominantes del ensamble de aves presente en el Centro Putemún, pertenecen a los gremios tróficos de aves piscívoras, omnívoras y depredadoras de peces e invertebrados marinos, respectivamente. Las presas que conforman a dichos grupos alimenticios poseen una fuerte asociación con las actividades de mitilicultura (Souza-Conceição et al. 2003), en donde peces como el pejegallo Callorhinchus callorhynchus (Linnaeus, 1758), el pejerrey Odontesthes regia (Humboldt, 1833) y el róbalo Eleginops maclovinus (Valenciennes, 1830) son considerados depredadores de 
Tabla 1. Abundancia relativa mensual y total, riqueza específica mensual observada y esperada del ensamble de aves asociadas al Centro Putemún, Chiloé. Se indica el grupo alimenticio (G.A.) de cada ave ( $P=$ piscívoro, $\mathbf{0}=$ omnívoro, $\mathbf{P I}=$ peces e invertebrados, $\mathbf{I}=$ invertebrados, $\mathbf{H}=$ herbívoro) / Relative monthly abundance and total abundance, observed and expected monthly specific richness of birds assemblages associated to Putemún Center, Chiloé. This table indicates the trophic group (G. A.) of each bird ( $P=$ piscivorous, $O=$ omnivorous, $\mathrm{PI}=$ fish and invertebrates, $\mathrm{I}=$ invertebrates, $\mathrm{H}=$ herbivorous)

\begin{tabular}{|c|c|c|c|c|c|c|c|c|c|c|c|c|}
\hline Especies & Sep-08 & Oct- 08 & Nov-08 & Dic-08 & Ene-09 & Feb-09 & Abr-09 & Jun-09 & Jul-09 & Ago-09 & Total & G.A. \\
\hline Phalacrocorax brasiliamus & 128 & 137 & 155 & 75 & 264 & 111 & 164 & 111 & 229 & 394 & 1768 & $\mathrm{P}$ \\
\hline Larus dominicanus & 12 & 10 & 12 & 10 & 19 & 26 & 81 & 30 & 41 & 15 & 256 & $\mathrm{O}$ \\
\hline Phalacrocorax atriceps & 2 & 4 & 3 & 2 & 15 & 29 & 79 & 48 & 26 & 19 & 227 & PI \\
\hline Sterna hirundinacea & 2 & 4 & 30 & 58 & 38 & 16 & 17 & 0 & 0 & 5 & 170 & PI \\
\hline Pelecanus thagus & 2 & 6 & 30 & 0 & 0 & 0 & 12 & 1 & 4 & 6 & 61 & $\mathrm{P}$ \\
\hline Chroicocephalus maculipennis & 0 & 0 & 4 & 1 & 3 & 6 & 2 & 4 & 1 & 4 & 25 & PI \\
\hline Tachyeres pteneres & 4 & 2 & 2 & 2 & 2 & 2 & 3 & 0 & 0 & 1 & 18 & PI \\
\hline Podiceps occipitalis & 0 & 0 & 0 & 0 & 0 & 0 & 0 & 4 & 6 & 7 & 17 & PI \\
\hline Sula variegata & 0 & 0 & 0 & 0 & 0 & 0 & 0 & 0 & 0 & 13 & 13 & PI \\
\hline Nycticorax nycticorax & 0 & 1 & 1 & 1 & 0 & 3 & 0 & 1 & 1 & 0 & 8 & PI \\
\hline Rollandia rolland & 1 & 0 & 0 & 6 & 0 & 0 & 0 & 0 & 0 & 0 & 7 & PI \\
\hline Leucophaeus pipixcan & 0 & 0 & 0 & 0 & 1 & 2 & 0 & 0 & 0 & 0 & 3 & PI \\
\hline Tachyeres patachonicus & 0 & 3 & 0 & 0 & 0 & 0 & 0 & 0 & 0 & 0 & 3 & I \\
\hline Milvago chimango & 1 & 1 & 0 & 0 & 1 & 0 & 0 & 0 & 0 & 0 & 3 & $\mathrm{O}$ \\
\hline Phalacrocorax gaimardi & 0 & 2 & 0 & 0 & 0 & 0 & 0 & 0 & 0 & 0 & 2 & $P$ \\
\hline Podiceps major & 0 & 0 & 0 & 1 & 0 & 0 & 0 & 0 & 0 & 0 & 1 & PI \\
\hline Fulica armillata & 0 & 0 & 0 & 0 & 0 & 0 & 0 & 1 & 0 & 0 & 1 & $\mathrm{H}$ \\
\hline Abundancia & 152 & 170 & 237 & 156 & 343 & 195 & 358 & 200 & 308 & 464 & & \\
\hline Riqueza observada & 8 & 10 & 8 & 9 & 8 & 8 & 7 & 8 & 7 & 9 & & \\
\hline Riqueza esperada & 7,99 & 9,89 & 7,97 & 8,9 & 7,99 & 7,99 & 6,99 & 7,86 & 6,95 & 8,99 & & \\
\hline
\end{tabular}

mitílidos en cultivo (Medina-Vogel 1989). Así también, existen invertebrados marinos epifaunales de mitílidos cultivados e incrustantes (i.e., fouling) de las estructuras utilizadas para el cultivo, generando dificultades de manejo para los productores (Kirk et al. 2007).

El alto porcentaje de aves piscívoras asociadas al Centro Putemún, sugiere que estas aves se alimentan de los peces que depredan los mitílidos en cultivo, generando un potencial beneficio para la industria mitilicultora al controlar dichas poblaciones de peces. Lo anterior solo puede ser validado mediante trabajos de campo que permitan conocer la dieta de las aves asociadas a esta actividad productiva. En apoyo a lo anterior, en centros mitilicultores de Canadá se ha registrado que la acción depredadora de patos marinos permitió disminuir hasta un 97\% la epifauna de los mitílidos cultivados (Kirk et al. 2007). A su vez, en mitiliculturas de Irlanda se han observado gaviotas depredando los organismos incrustantes desde las estructuras utilizadas para el cultivo (Roycroft et al. 2007). Esta relación entre aves marinas y mitilicultores ha sido considerada como una interacción mutuamente beneficiosa, en la cual como pocas veces, el desarrollo de una industria ha generado efectos positivos sobre la vida silvestre (Zydelis et al. 2009). Aún así, en centros mitilicultores de países del hemisferio norte (e.g., Escocia, Canadá y Estados Unidos) varias especies de patos marinos son consideradas como importantes depredadores de los mitílidos cultivados (Ross \& Furness 2000). A diferencia de dichos países, Chile presenta solamente al pato quetru no volador Tachyeres pteneres (Förster, 1844), considerado como un anátido estrictamente marino (Schlatter \& Simeone 1999), sobre el cual se estimó que su depredación sobre mitílidos cultivados no superó el 0,18\% de la producción total para un centro mitilicultor en Chiloé (Medina-Vogel 1989). Pese a ello, la recomendación internacional es que las abundancias de patos marinos en sitios con mitilicultura se deben registrar con regularidad para determinar si es creciente la tendencia temporal (Ross \& Furness 2000).

Las actividades de mitilicultura generan alteraciones significativas al paisaje costero (e.g., ingreso de estructuras flotantes) (Branco et al. 2001). Durante estudios comparativos entre sitios con y sin mitilicultura en las costas de Irlanda, no se observaron diferencias significativas entre la riqueza y diversidad de los ensambles de aves presentes para cada sitio, aunque los 
sitios sin actividad mitilicultora presentaron generalmente mayor diversidad de aves que los sitios con cultivo (Roycroft et al. 2004). Para el caso de Chiloé, en el Centro Putemún, sitio con mitilicultura, se registraron 17 especies de aves acuáticas, y en el sector conocido como Humedal de Putemún $\left(42^{\circ} 26^{\prime} \mathrm{S} ; 7^{\circ} 44^{\prime} \mathrm{O}\right)$, sitio sin mitilicultura, distante a $3 \mathrm{~km}$ al norte del Centro Putemún, se registraron 20 especies de aves acuáticas presentes en el cuerpo de agua, durante la misma temporada de estudio (CG. Suazo obs. pers.). La escasez de información en Chile impide generar un mayor análisis y discusión sobre los efectos que potencialmente generan las actividades de mitilicultura sobre las comunidades de aves acuáticas.

Se ha considerado a las estructuras de cultivo utilizadas por la industria mitilicultora (e.g., boyas flotantes y plataformas) un nuevo sustrato que permite el descanso, acicalamiento y nidificación de las aves, ya que brindan sitios de resguardo ante la presencia de depredadores y perturbación humana, para aves marinas y playeras (Branco et al. 2001, Andres et al. 2007, Roycroft et al. 2007). Los altos niveles de excremento aportados a lo largo del tiempo por las aves marinas que utilizan estos 'sustratos', sugiere una contribución a la productividad primaria de las zonas utilizadas por la mitilicultura (Branco et al. 2001) y para la salud pública esto es relevante, ya que en zonas mitilicultoras de Chiloé, se han encontrado patógenos y agentes parasitarios en excrementos de aves como el yeco y la gaviota dominicana (Torres et al. 1991), los cuales pueden contagiar a los humanos que consumen mitílidos crudos (Branco et al. 2001). Esta potencial amenaza para la salud humana se suma a las necesidades de investigación que poseen las interacciones de esta industria con el medio ambiente en el cual se desarrolla.

Finalmente, es recomendable crear un plan de monitoreo sobre los ensambles de aves marinas asociadas a centros mitilicultores ubicados en distintas localidades geográficas del país y en diferentes niveles de producción y etapas de proceso. Por lo anterior, es primordial evaluar las poblaciones de fauna silvestre que interactúan con este tipo de cultivo en cuanto a su disponibilidad de biomasa como alimento, así como en el uso de la infraestructura dispuesta por esta industria. A su vez, es urgente generar investigación que relacione los potenciales impactos del cultivo intensivo de mitílidos sobre otros componentes del ecosistema marino del sur de Chile y su productividad.

\section{Agradecimientos}

A la Dirección de Investigación de la Universidad de Los Lagos por financiar los costos de esta publicación. A Patricio Salas, Gastón Vidal, Luis Figueroa, P. Francisco Cárcamo, Marina Oyarzún, Heraldo Contreras, Alejandra Montaner y César Loncón, todos pertenecientes al CTPAPutemún (IFOP). JA. Cursach y CN. Tobar agradecen al Programa de Magíster en Ciencias de la Universidad de Los Lagos, en especial a la beca Nibaldo Bahamonde. CG. Suazo agradece el apoyo de CONICYT, al Centro de Investigación en Ecosistemas de la Patagonia (CIEP) y a la Universidad de Extremadura (España). A dos revisores anónimos de la RBM\&O.

\section{LITERATURA CITADA}

Andres BA, JA Johnson \& J Valenzuela. 2007. Whimbrels use novel high tide roosts during the contranuptial season in southern Chile. Wader Study Group Bulletin 112: 67-68.

Arriagada A. 2004. Efecto del cultivo de salmonídeos sobre la estructura comunitaria de aves en la región de Los Lagos, Chile. Seminario de Título, Departamento de Acuicultura y Recursos Acuáticos, Universidad de Los Lagos, Osorno, 96 pp.

Bertellotti M \& P Yorio. 1999. Spatial and temporal patterns in the diet of the kelp gulls in Patagonia. The Condor 101: 790-798.

Bibby C, N Burgess, D Hill \& S Mustoe. 2000. Bird census techniques, 302 pp, Academic Press, London.

Branco JO, JR Reuter-Braun \& JR Verani. 2001. Seasonal variation in the abundance of seabirds in areas of mariculture. Brazilian Archives of Biology and Technology 44: 395-399.

Buschmann AH, VH Riquelme, MC Hernández-González, D Varela, JE Jiménez, LA Henríquez, PA Vergara, $R$ Guínez \& L Filún. 2006. A review of the impacts of salmonid farming on marine coastal ecosystems in the southeast Pacific. ICES Journal of Marine Science 63: 13381345.

Casaux R, M Favero, N Coria \& P Silva. 1997. Diet of the imperial cormorant Phalacrocorax atriceps: comparison of pellets and stomach contents. Marine Ornithology 25: 1-4.

Ferrari S, B Alegre \& P Gandini. 2004. Dieta del cormorán imperial (Phalacrocorax atriceps) en el sur de Santa Cruz (Patagonia, Argentina). Ornitología Neotropical 15: 103110.

González-Gajardo A, P Victoriano \& R Schlatter. 2009. Waterbird assemblages and habitat characteristics in wetlands: influence of temporal variability on specieshabitat relationships. Waterbirds 32: 225-233. 
Gosztonyi A \& L Kuba. 1998. Fishes in the diet of the imperial cormorant Phalacrocorax atriceps at Punta Lobería, Chubut, Argentina. Marine Ornithology 26: 59-61.

Jaksic F. 2004. El Niño effects on avian ecology: lessons learned from the southeastern Pacific. Ornitología Neotropical 15(Suppl.): 61-72.

Kirk M, D Esler \& S Boyd. 2007. Morphology and density of mussels on natural and aquaculture structure habitats: implications for sea duck predators. Marine Ecology Progress Series 346: 179-187.

Ludynia K, S Garthe \& G Luna-Jorquera. 2005. Seasonal and regional variation in the diet of the kelp gull in northern Chile. Waterbirds 28: 359-365.

McAleece N, PJD Lambshead \& GLJ Paterson. 1997. Biodiversity Pro. The Natural History Museum, London. [en línea] <http://www.sams.ac.uk/>

Medina-Vogel G. 1989. Contribución a la ecología del pato quetru no volador (Tachyeres pteneres) y la relación de éste con la mitilicultura en Yaldad, Chiloé insular, 87 pp. Seminario de Título, Facultad de Ciencias, Universidad Austral de Chile, Valdivia.

Petracci P, L La Sala, G Aguerre, CH Pérez, N Acosta, M Sotelo \& C Pamparana. 2004. Dieta de la gaviota cocinera (Larus dominicanus) durante el período reproductivo en el estuario de Bahía Blanca, Buenos Aires, Argentina. Hornero 19: 23-28.

Quintana F, P Yorio, N Lisnizer, A Gatto \& G Soria. 2004. Diving behavior and foraging areas of the neotropic cormorant at a marine colony in Patagonia, Argentina. Wilson Bulletin 116: 83-88.

Remsen JV, CD Cadena, A Jaramillo, M Nores, JF Pacheco, MB Robbins, TS Schulenberg, FG Stiles, DF Stotz \& KJ Zimmer. 2011. A classification of the bird species of South America. American Ornithologists’ Union. [en línea] <http://www.museum.lsu.edu/ Remsen/SACCBaseline.html>
Ross B \& R Furness. 2000. Minimising the impact of eider ducks on mussel farming, 54 pp. University of Glasgow, Glaswog.

Roycroft D, T Kelly \& L Lewis. 2004. Birds, seals and the suspension culture of mussels in Bantry Bay, a non-seaduck area in Southwest Ireland. Estuarine Coastal and Shelf Science 61: 703-712.

Roycroft D, TC Kelly \& LJ Lewis. 2007. Behavioural interactions of seabirds with suspended mussel longlines. Aqualculture International 15: 25-36.

Schlatter R \& A Simeone. 1999. Estado del conocimiento y conservación de las aves en mares chilenos. Estudios Oceanológicos 18: 25-33.

Silva MP, R Bastida \& C Darrieu. 2000. Dieta de la gaviota cocinera (Larus dominicanus) en zonas costeras de la provincia de Buenos Aires, Argentina. Ornitología Neotropical 11: 331-339.

Souza-Conceição J, M Castro-Silva, G Huergo, G Soares, A Marenzi \& G Manzini. 2003. Associação da ictiofauna capturada através de rede de emalhe com o cultivo de mexilhões da enseada de Armação do Itapocoroy, em Penha (Santa Catarina-Brasil). Boletim do Instituto de Pesca 29: 117-121.

Torres P, E Ruíz, W Gesche \& A Montefusco. 1991. Gastrointestinal helminths of fish-eating birds from Chiloe island, Chile. Journal of Wildlife Diseases 27: 178-179.

Vilina Y, H Cofré \& C Pizarro. 2006. Reporte final aves acuáticas de Chile, $40 \mathrm{pp}$. Waterbird Conservation of the Americas, BirdLife International. [en línea] <http:// www.birdlife.org/action/science/species/waterbirds/ waterbirds_pdf/waterbirds_report_chile_2006.pdf>

Zydelis R, D Esler, M Kirk \& W Boyd. 2009. Effects of offbottom shellfish aquaculture on winter habitat use by molluscivorous sea ducks. Aquatic Conservation: Marine and Freshwater Ecosystems 19: 34-42.

Recibido el 24 de noviembre de 2010 y aceptado el 12 de enero de 2011 